\title{
Comparative Evaluation of the Regular Ovitrap vs an Innovated Larvitrap for Aedes Entomological Surveillance in Tapachula
}

\author{
Walter Eduardo Quezada-Yaguachi', Americo D. Rodriguez ${ }^{1 \dagger}$, Francisco Solís-Santoyo ${ }^{1}$, \\ Alma D. Lopez-Solis ${ }^{1}$, William C. Black IV ${ }^{2}$, Karla Saavedra-Rodriguez ${ }^{2}$, \\ Diego Morales-Viteri ${ }^{3}$, Patricia Penilla-Navarro ${ }^{{ }^{*}}$ \\ ${ }^{1}$ Centro Regional de Investigación en Salud Pública, Instituto Nacional de Salud Pública, Tapachula, Mexico \\ ${ }^{2}$ Arthropod Borne and Infectious Diseases Laboratory, Department of Microbiology, Immunology and Pathology, College of \\ Veterinary Medicine and Biomedical Sciences, Colorado State University, Fort Collins, USA \\ ${ }^{3}$ Centro de Referencia Nacional de Vectores, Instituto Nacional de Investigación en Salud Pública, Quito, Ecuador \\ Email: *penilla@insp.mx
}

How to cite this paper: Quezada-Yaguachi, W.E., Rodriguez, A.D., Solís-Santoyo, F., Lopez-Solis, A.D., Black IV, W.C., Saavedra-Rodriguez, K., Morales-Viteri, D. and Penilla-Navarro, P. (2022) Comparative Evaluation of the Regular Ovitrap vs an Innovated Larvitrap for Aedes Entomological Surveillance in Tapachula. Advances in Entomology, 10, 77-84.

https://doi.org/10.4236/ae.2022.101006

Received: October 21, 2021

Accepted: January 3, 2022

Published: January 6, 2022

Copyright $\odot 2022$ by author(s) and Scientific Research Publishing Inc. This work is licensed under the Creative Commons Attribution International License (CC BY 4.0).

http://creativecommons.org/licenses/by/4.0/

\begin{abstract}
Objective: The objective is to compare a regular ovitrap versus an innovated larvitrap for monitoring Aedes spp. populations. Materials and Methods: A total of 20 regular ovitraps and 20 innovated larvitraps were placed in pairs in 20 houses from the 5 de Febrero neighborhood in Tapachula, Chiapas, Mexico. The innovation consisted in the incorporation of a valve in the lower part of a half tire to drain the contents in a $2 \mathrm{~L}$ collection container. The evaluation was carried out during five weeks, collecting eggs and larvae from the ovitraps and innovated larvitraps, respectively. Positivity indexes and insectary production of adult Aedes spp. mosquitoes were compared by collection type. Results: Average positivity index for the five weeks period were $60 \%$ for ovitraps and $91.25 \%$ for innovated larvitraps. During the five weeks, $4043 \mathrm{Ae}$. aegypti and 703 Ae. albopictus adult mosquitoes were produced in the insectary from the eggs collected from ovitraps, while from innovated larvitraps were 9014 Ae. aegypti, 1205 Ae albopictus, and 15 Culex spp. Conclusion: Collection by the innovated larvitrap was more efficient, collecting 3.56 times more Ae. aegypti than with ovitraps, using approximately the same effort in time for replacing the filter paper from traditional $1 \mathrm{~L}$ ovitraps. Since the logistics for the storage and placement of larvitraps may still be a disadvantage in comparison with ovitraps, their use could be specifically intended in sentinel sites for mosquito population monitoring for entomological surveillance purposes.
\end{abstract}

${ }^{\dagger}$ Co-author Americo Rodriguez passed away a year ago. 


\section{Keywords}

Innovated Larvitrap, Ovitrap, Aedes, Entomological Surveillance

\section{Introduction}

Vector-borne diseases such as dengue, chikungunya and Zika are mainly transmitted by Aedes aegypti and represent an important problem of public health worldwide [1]. Since no vaccines or specific treatments are available, the use of insecticides is still the major component for the control of each of these diseases. The monitoring of Ae. aegypti populations is vital in the context of epidemiological surveillance including assessing susceptibility/resistance to insecticides, to see the impact of control measures [2].

This surveillance can be done by collecting adult mosquitoes, larvae, or eggs. In the context of insecticide resistance monitoring, the WHO recommends collecting mosquito larvae from the sites of interest to obtain a parental generation from which a first-generation can be used to conduct the bioassays [3]. For the surveillance, different types of larvitraps have been used, such as plastic containers, bamboo internodes, and tires, identifying the tires with the highest abundance of individuals [4].

However, the use of ovitraps to obtain eggs has proven to be extremely practical for epidemiological surveillance and therefore has been used for the monitoring of insecticide resistance by collecting eggs and obtaining adult mosquitoes from them [5]. On the other hand, the use of larvitraps has not been much implemented given the tedious to collect the larvae and pupae from the tires that are commonly adapted as larvitraps.

In this study, we are reporting the innovation and performance of a larvitrap that allows collecting in a fast and effective way immature stages of Aedes spp. mosquitoes.

\section{Materials and Methods}

The study was conducted in Tapachula, Chiapas, Mexico, where five blocks from the neighborhood 5 de Febrero were selected. Innovated larvitraps were built in the Centro Regional de Investigación en Salud Pública (CRISP) facilities using half of a car tire such as those previously reported [6]. The innovation of this type of larvitrap was the incorporation of a valve in the lower part of the half tire to drain the contents in a $2 \mathrm{~L}$ collection container (Figure 1), which ensured the collection of all the biological material contained in the larvitrap. This innovation also requires a shorter collection time compared to the use of pipetting to collect the material in a larvitrap without this modification.

Each innovated larvitrap, hanging at $1 \mathrm{~m}$ from the floor and with $3 \mathrm{~L}$ of water, was placed at least $1 \mathrm{~m}$ apart of an ovitrap (Figure 1), which was made of a black plastic container of $1 \mathrm{~L}$ capacity, internally lined with filter paper as recommended 


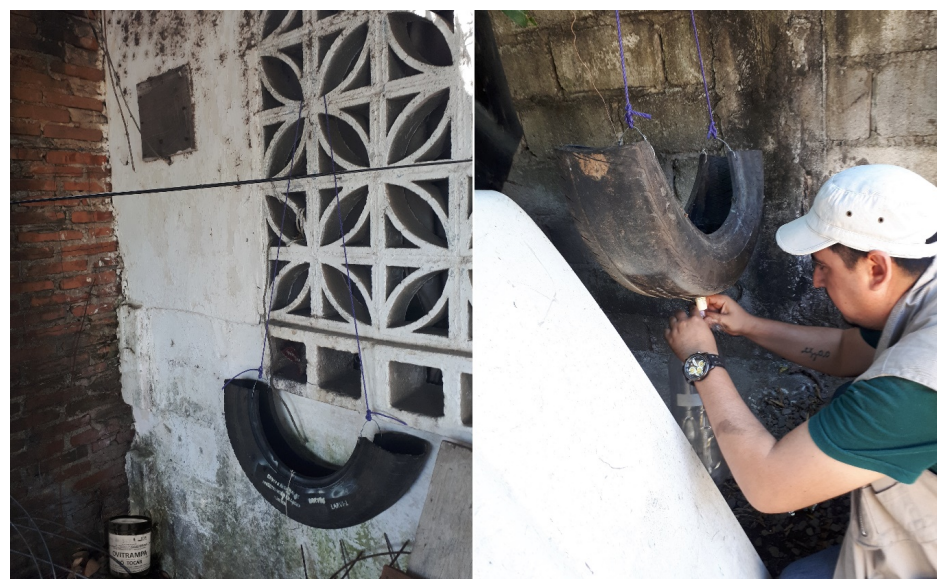

Figure 1. Left: ovitrap placed on the floor next to an innovated larvitrap (with a valve incorporated in the lower part of the half of a tire) that hangs $1 \mathrm{~m}$ from the ground, containing $3 \mathrm{~L}$ of water. Right: the content of an innovated larvitrap is drained into a $2 \mathrm{~L}$ collecting container, where all the larvae and pupae are collected.

by the National Vector Control Program from Mexico [7]. A total of 20 ovitraps and 20 innovated larvitraps were placed in the backyards of 20 houses in the selected study area.

The evaluation was carried out during five weeks from January to February 2018, collecting the first week in a traditional way by pipetting the innovated larvitraps, that is, without using the valve, in order to measure the collection time for comparison purposes with time employed when using the valve. During the remaining four weeks the drainage system was used. Eggs, larvae and pupae collected every 7 days were taken to CRISP and reared in the insectary under standardized conditions of temperature of $27^{\circ} \mathrm{C} \pm 2^{\circ} \mathrm{C}, 70 \%-80 \%$ humidity, and a 12:12 hour photoperiod.

The positivity indexes of ovitraps (for eggs), and innovated larvitraps (for larvae and pupae) were calculated using the formula: number of positive ovitraps or innovated larvitraps divided by the number of ovitraps or innovated larvitraps placed in the 20 houses, all multiplied by 100 . The production in the insectary of adult Aedes spp. mosquitoes were compared by type of collection and the effectiveness of ovitrap vs innovated larvitrap evaluated.

\section{Results}

\subsection{Positivity Indexes}

The positivity index for eggs for the ovitraps was calculated for each of the five weeks of the study resulting in $75 \%, 65 \%, 45 \%, 55 \%$, and $70 \%$, respectively (Figure 2), with an average of $60 \%$ for the five weeks period. For innovated larvitraps, positivity indexes for larvae were $85 \%, 95 \%, 90 \%, 95 \%$, and $100 \%$ (Figure 2), with an average of $91.25 \%$ for the five weeks period. Positivity index for pupae from the same innovated larvitraps were $40 \%, 95 \%, 65 \%, 70 \%$, and $25 \%$ (Figure 2), with an average of $67 \%$. 


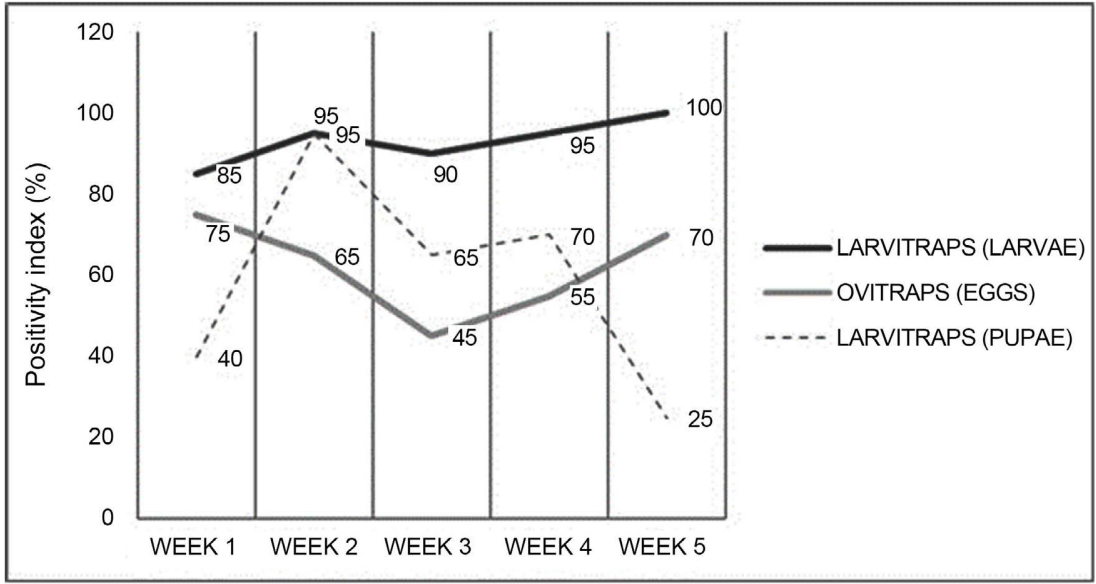

Figure 2. Positivity index for the ovitraps and the innovated larvitraps (week 1 was pipetted as traditional larvitrap) in the 5 de Febrero neighborhood, in Tapachula, Chiapas, Mexico.

\subsection{Productivity in the Offspring by Collection Method}

The mean of eggs collected by ovitrap $(n=20) \pm$ standard error is shown in Figure 3 for each of the five weeks, with 5707 eggs collected during the study. Weekly species proportion collected per ovitrap (Ae. aegyptivs Ae. albopictus) is shown in Figure 4. From these eggs collected during the five weeks, 4043 Ae. aegypti and 703 Ae. albopictus adult mosquitoes were recorded in the insectary during the study.

No eggs were attempted to be collected with innovated larvitraps. Innovated larvitrap mean larvae collected $(n=20) \pm$ standard error is shown in Figure 3 for each of the five weeks, with 10,984 larvae collected during the study. The collected proportion of Ae. aegypti in relation to Ae albopictus and Culex spp. in the 20 innovated larvitraps for each week is shown in Figure 4. In total $9014 \mathrm{Ae}$. aegypti, 1205 Ae. albopictus, and 15 Culex spp. adult mosquitoes were recorded in the insectary during the study.

\subsection{Effectiveness of Ovitrap vs Innovated Larvitrap}

The number of adults Ae. aegypti mosquitoes obtained from collections with innovated larvitraps exceeded 1.63,6.12, 5, and 1.5 times the number of specimens obtained through ovitraps during weeks 2 to 5 respectively with an average of 3.56 times, when the adapted drain system was used (Table 1). Finally, the effort in time used to collect the specimens by using pipettes was $20 \mathrm{~min}$ in average (week 1), while using the drain valve was $5 \mathrm{~min}$ in average, which is approximately the same effort in time for replacing the filter paper from traditional $1 \mathrm{~L}$ ovitraps.

\subsection{Effectiveness of Larvitrap (Pipetting in the Traditional Way) vs Innovated Larvitrap}

The number of adults Ae. aegypti mosquitoes obtained from collections of weeks 2 to 5 with innovated larvitraps exceeded 6.37, 12.5, 10.06, and 9.59 times, respectively the number of specimens obtained through traditional larvitrap (col- 
lecting using pipettes) in week one (Table 1). The innovated larvitrap on average collected 9.63 times more larvae than the traditional larvitrap, while the collection time was reduced from 20 to $5 \mathrm{~min}$.

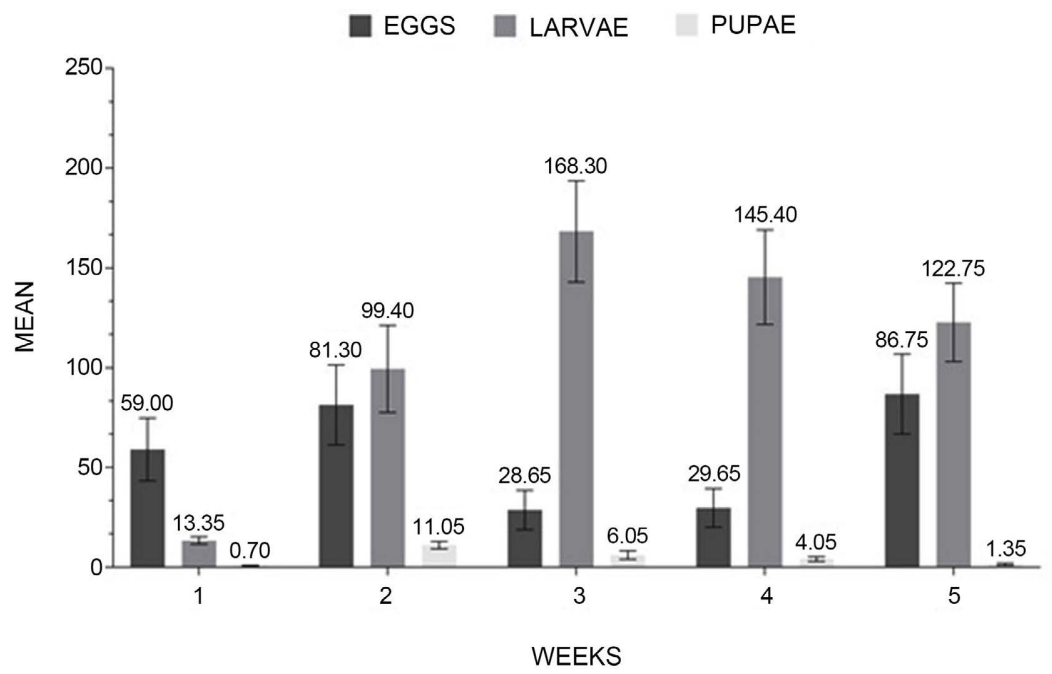

Figure 3. Mean \pm SE of eggs, larvae and pupae collected in ovitraps and innovated larvitraps per week (week 1 was pipetted as traditional larvitrap) in the 5 de Febrero neighborhood, in Tapachula, Chiapas, Mexico.

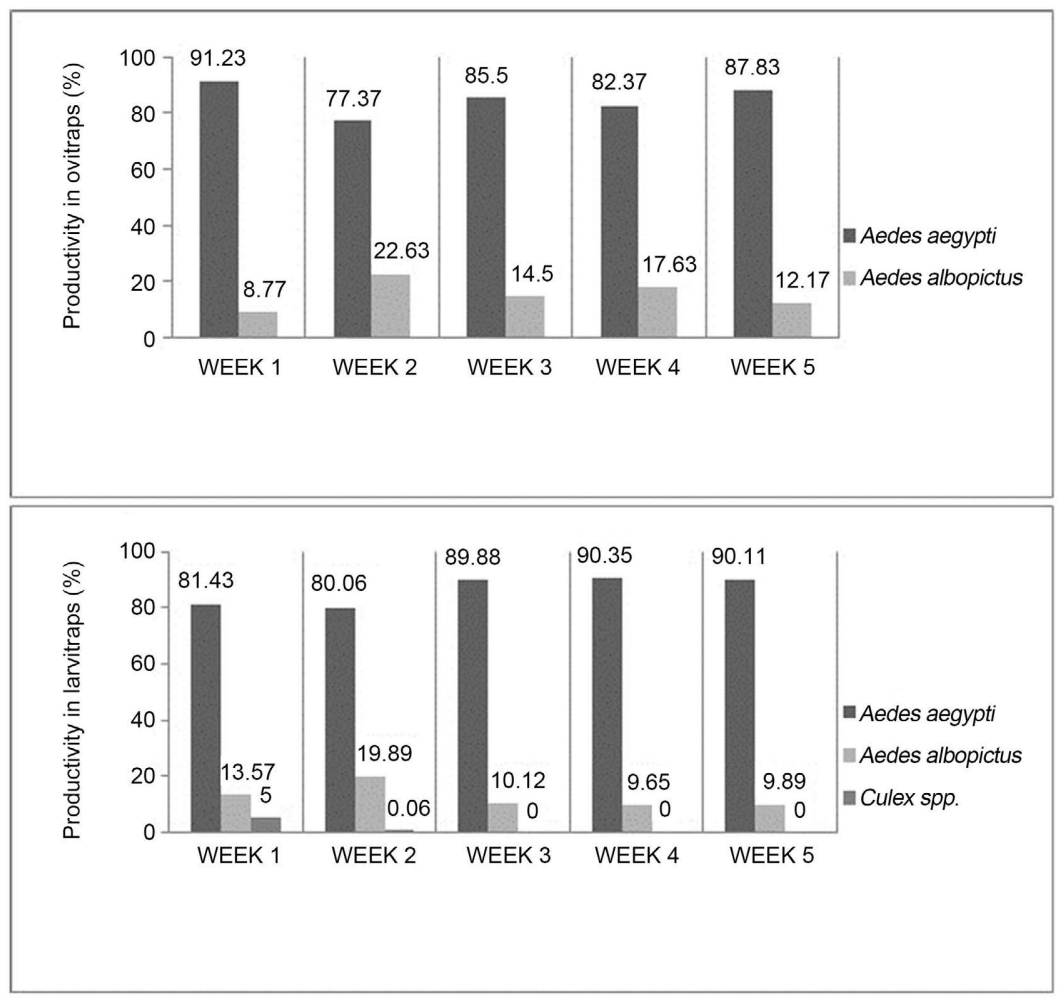

Figure 4. Above: proportion of Ae. aegypti vs Ae. albopictus offspring produced in the insectary from collections by ovitrap and; bellow: proportions of Ae. aegypti, Ae. albopictus and Culex spp. offspring produced in the insectary from collections by innovated larvitraps (week 1 was pipetted as traditional larvitrap) in a neighborhood from Tapachula, Chiapas, Mexico. 
Table 1. Offspring total Aedes aegypti adult mosquitoes produced in the insectary by collection type undertaken in a neighborhood of Tapachula, Chiapas, Mexico.

\begin{tabular}{ccccccc}
\hline Collection tool & Week 1* & Week 2 & Week 3 & Week 4 & Week 5 & Total \\
\hline Ovitraps & 780 & 889 & 466 & 458 & 1450 & 4043 \\
Innovated larvitraps & 228 & 1453 & 2852 & 2294 & 2187 & 9014 \\
\hline
\end{tabular}

${ }^{\star}$ Without the drainage system.

\section{Discussion and Conclusion}

The methods for collecting Ae. aegypti are used mainly for the elaboration of indexes for epidemiological stratification, entomological risk and the effectiveness of control measures [8]. The two major methods consist in the collection of eggs, or larvae and pupae by means of ovitraps or larvitraps, respectively. They are also very useful to collect mosquitoes to test for insecticide resistance, since not always the collection from natural or man-made occurring breeding sites is possible. Ovitraps have evolved from the use of a wooden stick into a $1 \mathrm{~L}$ water container to the actual "traditional" ovitraps, which include the use of filter paper placed on the inner wall of the container [7]. Larvitraps on the contrary, have been implemented by the use of half of a used car tire filled with water and its evolution or innovation have been very little [9]. In the present study, we compared the effectiveness of innovated larvitraps against traditional ovitraps based on the positivity index and the abundance of adult mosquitoes produced in the insectary from the collections using both methods.

Traditional vs innovated larvitrap had a disadvantage in the numbers of larvae collected as well as in the time for collection. The innovated larvitrap on average collected 9.6 times more larvae than the traditional larvitrap, while the collection time was reduced from 20 to $5 \mathrm{~min}$. Furthermore, the innovated larvitrap had a higher positivity index and higher offspring capacity from the immature biological material collected compared to ovitraps, in almost the entire period of the entomological monitoring. Studies carried out by Lima et al. (1989) [10] comparing different types of traps, showed larvitraps as more attractive than ovitraps. They suggested that the higher surface and volume of water in the larvitrap influenced oviposition, resulting in higher efficiency. Other factors like the color and the rubber composition of the tire, could provide more stable environmental conditions such as water temperature, that favor the oviposition and development of Ae. aegypti larvae.

Under different circumstances, either methodological or environmental, the surveillance tools for Ae aegypti monitoring has to be adequate to the established aims, as well as supported by efficiency, operating time and cost [11]. The ideal will always be to have a specific, economical and sensitive technique to sample any mosquito population. Ovitraps represent a good tool for the early detection of dengue and yellow fever vectors [12]. Similarly, ovitraps were positive even in the presence of natural breeding sites and presented superior efficiency to larvitraps [13]. In our study, we did not register the presence of natural 
breeding sites in the study area, however, the conditions of the selected houses were similar to each other, and so the presence of other breeding sites in addition to ovitraps and larvitraps could have been similar. Despite this, larvitraps proved to be much more attractive than ovitraps. Moreover, ovitraps and innovated larvitraps were placed in a paired way, so the attraction of each method was in some way under test in the design. However, a study where local mosquito populations are at very low levels could indicate which method is more sensitive to detect low Ae. aegypti populations.

Larvitraps seem to have a great potential even representing an economic method for the detection and collection of Ae. aegypti larvae and pupae. Considering these advantages, it is suggested that the operative use of innovated larvitraps could result in a sensitive, rapid and efficient technique for Ae. aegypti monitoring. However, the logistics for the storage and placement of innovated larvitraps may still be a disadvantage in comparison with ovitraps, nevertheless, their use could be specifically intended in sentinel sites for population monitoring after an intervention by the control programs, including collections of biological material for insecticide resistance testing.

Finally, in the context of a pandemic such as the COVID-19, social distancing which is one of the most recommended measures along with facemask use could prevent the placement of ovitraps inside houses. While placing them outside the houses, could be impractical because of the size of the traps and the risk to be stolen for the potential use of the $1 \mathrm{~L}$ capacity containers for other purposes. The innovated larvitraps on the other hand, because of the size and less attractive appearance, could represent the best choice to monitor mosquito populations under these circumstances, since are less likely to be stolen. The above-mentioned assessments need to be tested in the field.

\section{Acknowledgements}

Project Supported by the Sectorial Fund for Research in Health and Social Security.

\section{Conflicts of Interest}

The authors declare no conflicts of interest regarding the publication of this paper.

\section{References}

[1] Ramos-Castañeda, J., Barreto dos Santos, F., Martínez-Vega, R., Galvão de Araujo, J.M., Joint, G. and Sarti, E. (2017) Dengue in Latin America: Systematic Review of Molecular Epidemiological Trends. PLoS Neglected Tropical Diseases, 11, 1-24. https://doi.org/10.1371/journal.pntd.0005224

[2] Morato, V.C.G., et al. (2005) Infestation of Aedes aegypti Estimated by Oviposition Traps in Brazil. Revista de Saude Publica, 39, 553-558.

https://www.scielo.br/scielo.php?script=sci_arttext\&pid=S0034-89102005000400006 \&lng $=$ pt\&nrm $=$ iso 
https://doi.org/10.1590/S0034-89102005000400006

[3] World Health Organization (2016) Entomological Surveillance for Aedes spp. in the Context of Zika Virus: Interim Guidance for Entomologists. World Health Organization. https://apps.who.int/iris/handle/10665/204624

[4] Almeida, J.F., Belchior, H.C.M., Ríos-Velásquez, C.M. and Pessoa, F.A.C. (2020) Diversity of Mosquitoes (Diptera: Culicidae) Collected in Different Types of Larvitraps in an Amazon Rural Settlement. PLoS ONE, 15, e0235726.

https://doi.org/10.1371/journal.pone.0235726

[5] Deming, R., Manrique-Saide, P., Medina Barreiro, A., Cardenã, E.U.K., Che-Mendoza, A., Jones, B., Liebman, K., Vizcaino, L., Vazquez-Prokopec, G. and Lenhart, A. (2016) Spatial Variation of Insecticide Resistance in the Dengue Vector Aedes aegypti Presents Unique Vector Control Challenges. Parasites \& Vectors, 9, Article No. 67. https://doi.org/10.1186/s13071-016-1346-3

[6] Suárez Ramírez, N. and Colás Bonne, M. (2008) Afectación de la sensibilidad del sistema de vigilancia entomológica mediante larvitrampas en un área de salud. Medisan, 12, 9 p. https://www.redalyc.org/pdf/3684/368445249011.pdf

[7] Centro Nacional de Programas Preventivos y Control de EnfermedadesCENAPRECE (2016). Guías Operativas para el Control de Vectores.

https://www.gob.mx/salud/cenaprece/documentos/guias-operativas-de-arbovirosis

[8] Paho, P.H. (2019) Handbook for Integrated Vector Management in the Americas. https://iris.paho.org/handle/10665.2/51759

[9] Valdés Miró, V., Reyez Arencibia, M., Bandomo Abreu, N., Leyva Silva, M. and MaruqettiFernández, M.delC. (2018) Evaluación de las larvitrampas como método de vigilancia de Aedes aegypti (Diptera:Culicidae) y otros culícidos. Revista Cubana de Medicina Tropical, 70, 2007-2010.

http://scielo.sld.cu/scielo.php?script $=$ sci_arttext\&pid $=$ S0375-07602018000300002

[10] Lima, M.M., et al. (1989) Estudo comparativo da eficácia de armadilhas para Aedes aegypti. Cadernos de Saúde Pública, 5, 143-150.

https://doi.org/10.1590/S0102-311X1989000200002

[11] Furlow, B. and Young, W. (1970) Larval Surveys Compared to Ovitrap Surveys for Detecting Aedes and Aedes triseratus. Mosquito News, 30, 468-470. https://www.biodiversitylibrary.org/content/part/JAMCA/MN_V30_N3_P468-470.pdf

[12] Chadee, D.D. (1990) Métodos de evaluación de la población de Aedes aegypti y tratamientos con insecticidas en una población de Trinidad, Antillas. Pan American Journal of Public Health, 109, 350-359. https://iris.paho.org/handle/10665.2/16704

[13] Marques, C.C., Marques, G.R., de Brito, M., dos Santos Neto, L.G., Ishibashi, V.C. and Gomes, F.A. (1993) Estudo comparativo de eficácia de larvitrampas e ovitrampas para vigilância de vetores de dengue e febre amarela. Revista de Saude Publica, 27, 237-241. https://doi.org/10.1590/S0034-89101993000400002 